(REVIEW ARTICLE)

\title{
Immunization strategies in dental caries: Scopes and limitations
}

\author{
Paola Michelle Cantos Tello ${ }^{1, *}$, Gabriela Cecibel Torres Tamayo ${ }^{1}$, Hugo Alejandro Abad Ortiz ${ }^{1}$ and María de \\ Lourdes Rodríguez Coyago 2,3,* \\ ${ }^{1}$ Universidad de Cuenca, Facultad de Odontología, Tenth grade students, Cuenca-Ecuador. \\ 2 Universidad de Cuenca, Facultad de Odontología, Chair of Semiology and Diagnostic Clinic. Cuenca-Ecuador. \\ ${ }^{3}$ Universidad de Buenos Aires, Institute for Research in Microbiology and Parasitology (IMPaM). Buenos Aires-Argentina.
}

Publication history: Received on 28 September 2020; revised on 03 October 2020; accepted on 06 October 2020

Article DOI: https://doi.org/10.30574/wjarr.2020.8.1.0361

\begin{abstract}
Dental caries is an infectious disease, multifactorial, and considered a global public health problem. Of the cariogenic microbiota, Streptococcus mutans concentrated for years as the main etiological agent of dental caries; leading the scientific community to consider it a target of immunization strategies. However, recent studies discovered a polymicrobial ecosystem with wide variability in dental caries, and $S$. mutans would represent only a small part of the bacterial community, being found in less than $1 \%$ in the different types of carious lesions. Several studies have been carried out that seek to control both the appearance and the advance of cavities, for which, vaccines have been developed in two ways: passive immunization and active immunization. Both types of immunization target antigens expressed on the cell surface of the microorganism. In this study, research on the subject published in the last twenty years is reviewed, in order to establish the current state of this field, and expose its scope and limitations when it comes to meeting the objective of reducing the incidence of caries, which, according to world load studies is still high.
\end{abstract}

Keywords: Dental caries; Streptococcus mutants; Vaccines; Passive immunity; Active immunity.

\section{Introduction}

Dental caries is a non-classical, localized infectious disease of multifactorial etiology resulting from the interaction of three main factors: a susceptible host, an unbalanced or dysbiotic microbe, and a fermentable substrate [1]. When interacting for a certain time, these factors will lead to the progressive demineralization of the dental hard tissue [2].

The cariogenic capacity of the bacteria involved in this process is determined by their ability to adhere to the tooth structure, their aciduricity, understood as their resistance to low $\mathrm{pH}$, and their acidgenicity, that is, their ability to produce lactic acid from the sugar in the food, resulting in the destruction of the hard tissues of the tooth [3]. Within the bacterial component, the main microorganisms classically associated with the carious process have been Streptococcus mutans and Streptococcus sobrinus [1]. Their main virulence factors have been related to the adherence of these bacteria to the dental structure, through surface and metabolism proteins, such as Ag I / II antigen (PAc) and glucosyltransferase (GTF), respectively. Which will promote, directly or indirectly, the adhesion and colonization of these microorganisms $[4]$.

This pathology is difficult to control given its polymicrobial nature, which is widely heterogeneous among individuals, types of caries and dentition [5,6]. Several prevention strategies in dental caries have been proposed, in addition to mechanical and chemical plaque removal measures [7]. The probiotic and prebiotic systems are aimed at modulating oral biofilm and mucosal-associated immunity; and several studies support the use of these systems to modulate plaque composition towards an increase in non-pathogenic commensal bacterial species and a decrease in the count of

\footnotetext{
* Corresponding author: Paola Michelle Cantos Tello

Universidad de Cuenca, Facultad de Odontología, Tenth grade students, Cuenca-Ecuador.
} 
cariogenic species such as $S$. mutans, maintaining a healthy and balanced ecosystem [8-10]. However, Simon-Soro and colleagues suggest that current clinical trials with probiotic treatment have not been sufficiently effective in reducing caries rates, which may be associated with the use of species unique to this type of approach. According to Simon-Soro et al. (2015), there is a need for future metatranscriptomic, proteomic or metabolomic approaches that allow the identification of molecules that may interfere with the initiation and progression of dental caries, and thus avoid the complex polymicrobial nature of this pathology [11]. Indeed, prevention strategies directed at specific bacterial species will not be fully effective, considering the bacterial diversity of dental caries [5].

Dental caries is an ancient disease, and even in the 21st century, it remains a global public health problem, affecting $60 \%$ to $90 \%$ of the child population and the vast majority of adults [4]. According to data from the study "The Global Burden of Disease 2015", untreated dental caries in permanent teeth was the most prevalent oral pathology (34.1\%), affecting 2.5 billion people worldwide. Meanwhile, the prevalence of untreated caries in deciduous teeth was $7.8 \%$, peaking in the 1-4 year age group worldwide. Meanwhile, caries in the permanent dentition was highest in the 15-19 year age group. Additionally, the highest prevalence of caries in permanent teeth was observed in Latin America (54.9\%), and no significant differences according to sex were reported worldwide [12]. In view of these alarming data, we ask ourselves if active or passive immunization strategies applied to dental caries can be an effective way to reduce the incidence of the disease by significantly decreasing the level of colonization of acidogenic and aciduric bacteria such as $S$. mutans.

Consequently, the objective of this study was to review the evidence available and published in the last 20 years regarding dental caries immunization strategies, as well as their advantages and disadvantages. Although several studies of dental caries vaccines have been published, only passive immunization has been widely tested in humans, while few clinical trials of active immunization exist $[1,4,13,14]$. Therefore, topics such as immunology and oral microbiota, which will be addressed in this review, could be useful for future research on the subject, aimed at developing a cost-effective vaccine to prevent this disease of high prevalence and incidence in the world.

\section{Material and methods}

A bibliographic review of different studies published in the scientific database PubMed, in the last 20 years, was carried out. The following keywords were used for the search: "Immunization AND dental caries", OR "Caries vaccine", OR "Streptococcus mutans vaccine", AND "Active immunization", AND "Passive immunization".

A total of 25 articles in the English language published between the years 2000 and 2020 were compiled, including: systematic reviews, meta-analyses, experimental studies, and clinical trials that fit the study objective. Narrative reviews were excluded. For the organization of the data, the program Microsoft Excel 2013 was used.

\section{Results and discussion}

Streptococcus mutans has a set of virulence factors that allow it to adhere and accumulate in dental biofilm. Three main groups of antigens associated with the surface of these microorganisms are involved in the process of adhesion and accumulation of this bacterium. These antigens are the main objectives for the development of the vaccine against caries: a) Glucosyltransferases (Gtfs), b) Antigenic adhesives I / II (Ag I / II) and c) Glucan-binding proteins (Gbp). One of the main virulence characteristics of $S$. mutans is its ability to produce Gtfs, enzymes that synthesize intracellular polysaccharides (ICP) and extracellular polysaccharides (ECP) from dietary sucrose. Thus, the various antigenic components against which immune responses are produced are adhesives, glucosyltransferases and glucan-binding proteins [15].

A variety of acidogenic microorganisms have been associated with different stages of dental caries, however, $S$. mutans has been uniformly identified in the white enamel demineralization spots, becoming a target for a caries vaccine. This bacterium has a cell wall with its respective adhesion-type antigens, and produces extracellular polysaccharides, which are the primary means of attachment to the tooth and accumulation to the biofilm $[3,16]$. Therefore, these components that participate in adhesion such as antigen I and II, the formation of glucans or cell wall synthesis, are immunogenic vaccine candidates that seek to inhibit caries formation. Research in this field aims at adaptive immunity against glucosyltransferase, an enzyme produced by $S$. mutans or $S$. sobrinus, which catalyzes the formation of alpha 1.3 and 1.6 glucans, exploring the immune effect of IgA and IgG antibodies [16-18].

In an animal study, the antigens administration increased the levels of IgA and anti-GTF salivary IgG, providing protection to caries in an experimental way $[16,19]$. In parallel, passive immune approaches have also demonstrated 
efficacy in experimental studies, in which, supplements of polyclonal IgG antibodies against GTF, GBP or monoclonal reagents that are specific for S.mutans cell surface antigens, have shown to reduce effectively the incidence of dental caries $[16,20]$.

Active immunization has been scarcely addressed in clinical studies, being studied by only one group of North American researchers, who evaluated the efficacy of a vaccine based on S. mutans GTF, administered by nasal and tonsillar routes, in different presentations of the antigen (only protein, or protein-lipid combinations). Although, the results of these studies have been encouraging, the number of participating subjects is limited, and the most important humoral immune response has been evidenced in nasal and salivary secretions, as long as the immunization is performed by intra-nasal route, and under lipid formulations [1,21].

\subsection{Passive immunization as a preventive strategy in dental caries}

Passive immunization results from the transfer of prefabricated antibodies that circulate in the body, and give specific protection. Experimentally successful passive vaccines for caries have taken many forms, including monoclonal mouse IgG, bovine or ape IgG and chicken IgY. They have also used bovine milk and serum generated from immunized cows to control S. mutans colonization [22].

Mitoma et al (2002), in their study on passive immunization with bovine milk, used immunized milk containing antibodies against PAcA- GB antigen, having as a result a clear suppression of caries development in rat models, being the first pre-clinical study that demonstrates favorable results of passive immunization with bovine milk, concluding that this immunized milk could be a viable option to control caries in humans [23]. In another study carried out by Shimazaki and cols (2001), in which they used immune milk from a cow challenged with PAcA-GB protein, protein resulting from the fusion between the alanine rich region of Pac (PAcA) and that binds to saliva, with the glucan binding domain (GB) of GTF; and on the other hand, it was used control group milk (Milk obtained from non-immunized cows). This study was carried out in humans, in which both groups were subjected to a two-week period of application of immune and non-immune milk respectively, resulting in a lower level of $S$. mutans in the group that received immunized milk [18].

Another method of passive immunization that has been tested in pre-clinical models, is based on using immunoglobulin Y (IgY) derived from chickens, as it has been reported that it can inhibit the binding of Streptococcus mutans to the acquired tooth film. The experimental study by Bachtiar et al. (2015), concludes that soy milk enriched with IgY and chitosan can reduce the number of $S$. mutans in the dental biofilm, and therefore prevent the development of caries. In the study by Bachtiar et al., the group of rats that received IgY plus chitosan-enriched soy milk had several S. mutans, twelve times lower than the control group. It was also estimated that IgY persisted in the saliva of these rats until 15 days after the feeding period. Although IgY can inhibit the binding of $S$. mutans to the dental film, this immunoglobulin is susceptible to changes in temperature and $\mathrm{pH}$ levels. Therefore, to improve its effect as a passive immunization, a preservative such as chitosan, a polysaccharide extracted from shell chitin of crustaceans, can be added. Several studies have shown that chitosan also has antibacterial effects, so it is expected that IgY and chitosan added to soy milk will decrease the formation of biofilms and the presence of cariogenic bacteria [24].

From the prefabricated and tested antibodies both at the pre-clinical and clinical level, the purified IgY from hen's egg yolk, has shown efficacy in reducing salivary levels of $S$. mutans. An example of this is the clinical trial of Nishihara et al. (2014), conducted in 64 healthy volunteers to evaluate the effects of two probiotic strains of Lactobacillus salivarius (administered in tablet form), versus a commercial product of IgY (Ovalgen DC), and xylitol on dental caries risk factors. In this study, the treatment with Ovalgen DC did not show significant differences, concerning the probiotic strains tested, in the capacity to reduce the salivary levels of S. mutans; while the treatment with xylitol showed inferiority in the measurement of this variable [13].

Nguyen Van Sa (2013), in his study on the specific antibody of egg yolk (Ovalgen DC) as a new supportive immunotherapy for dental caries, states that glycosyltransferase is an essential enzyme in the pathogenicity of $S$. mutans, and there are two types: 1) Glucosyltransferase-CF that produces soluble glucan, and 2) GlucosyltransferaseCA that produces insoluble glucan. The cell-associated glucosyltransferase (Glucosyltransferase-CA), is the main pathogenic factor of $S$. mutans. Insoluble glucan binds strongly to the surface of teeth and assists in the colonization of S. mutans. Egg yolk specific antibodies contain specific IgY against $S$. mutans glucosyltransferase. In this study, clinical trials were conducted, in which 4 healthy volunteers participated in a 2-phase test. In phase 1, a placebo mouthwash was used; and after 2 weeks, a mouthwash containing Ovalgen DC was used. Plaque scores were compared between the 2 phases, with the first group having a plaque score of 3, while the group treated with Ovalgen DC had a score of less than 2.8, according to the scale used in that study. The results showed a reduced number of $S$. mutans in the saliva of 
human volunteers treated with the commercial product Ovalgen DC, which did not affect the normal microbiota of the oral cavity [14].

Additionally, the effect of polyclonal immunoglobulin G (IgG) antibodies from egg yolk against whole cells, cell-free glucosyltransferase (CF-GTase) or cell-associated glucosyltransferase (CA-GTase) of Streptococcus mutans serotype c were examined. Purified egg yolk IgG showed strong inhibitory activity against CA-GTase but not against CF-GTase. Interestingly, CA-GTase-Specific IgG significantly inhibited the development of dental caries in rats that had been infected with $S$. mutans serotype c, but not CF-GTase-Specific IgG. In conclusion, the specific IgG for the CA-GTase of $S$. mutans inhibited the enzymatic activity that produces insoluble glucan from sucrose, resulting in inhibition of cell adhesion and decreased cariogenicity in S. mutans infected rats. IgG could be conveniently and stably obtained from eggs of hyperimmunized hens with purified CA-GTase and an adjuvant. Therefore, it is established that hens can provide a beneficial and economical source of antibodies for passive immunization [14].

Based on the previous exposition, the local passive immunization against $S$. mutans has been widely explored, using buccal topics and administration of antibodies derived from animals vaccinated with whole bacteria or components of these [25]. However, and according to Krugër et al., passive vaccines are less effective since they require more applications in high doses to achieve a persistent effect in time. To reduce this disadvantage, Krugër and collaborators built a series of expression vectors with inducible or constitutive promoters for the superficial expression or secretion of several antigens in bacteria of the genus Lactobacillus. Recently, a single-stranded antibody (scFv) generated from Guy's monoclonal antibody 13 directed against the SA I / II antigen of S. mutans, which was expressed on the surface of a bacterium of the genus Lactobacillus using an inducible promoter and demonstrating that it protects against the development of cavities when administered to rats. With this background, Krugër and his group constructed a new vector (pLP501-scFv-long anchor) containing a constituent promoter (Pldh) of the lactate dehydrogenase gene of Lactobacillus plantarum and encoding a Guy 13-derived scFv directed against the SA I/II adhesion antigen of S. mutans. According to Krugër et al., the bacteria of the genus Lactobacillus, which constitutively express this antibody, may not affect the number of $S$. mutans, but alter the stability of the biofilm. This influences the diffusion of the substrate (sucrose) or affects the release of bacterial metabolic products (such as lactic acid) within the biofilm, thus contributing to reducing the development of caries [25]. The idea of Krugër and his group is very innovative, since with his proposal, it is intended to passively immunize in a non-classical way, based on the administration of Lactobacillus plantarum strains transformed by genetic engineering to express in a persistent way and in high doses, a monoclonal antibody directed to a surface antigen of the cariogenic S. mutans, with the aim of minimizing its adherence to the acquired salivary film. Human studies are required to evaluate the efficacy and substantivity of this new and innovative biotechnological approach.

\subsection{Active immunization as a preventive strategy in dental caries}

The active immunization would be more effective and durable since it stimulates the participation or response of the host itself, and its stability in time through the immunological memory. As in the passive immunization, the active immunization has as objective target antigens expressed by Streptococcus mutans, as the adhesives of cellular surface, to be administered to the human host and induce the production of antibodies by its own immune system. Studies show that, developing antibodies against these adhesives prevent the adhesion of these bacterial species to the dental surface [4].

Cao et al. (2016), in a study that compared the levels of specific antibodies in children with different levels of S. mutans, mentions that the inhibition of GTF by the salivary secretory IgA (IgAs) protects against dental caries. GTF expresses an antigenic region, that is, a B cell epitope, which turns out to be a key target due to its mobility and flexibility. This unpreserved and unknown functional region of the GTF could be possible candidates for vaccine development. Additionally, Cao and collaborators evaluated the epitope region of the PAc antigen, with the objective of predicting possible targets for the vaccine. The group reported that PAc is the ideal antigen that induces the expression of inhibiting antibodies as a response of the host in human beings. Evidence has demonstrated greater effectiveness in the use of caries vaccines when combining epitopes of PAc and GTF. However, few studies have related these two epitopes in the construction of caries vaccines [4].

Ferreira et al. (2015), evaluated the induction of protective immunity against $S$. mutans utilizing a synthetic vaccine obtained from phosphate-binding protein (Psts), based on the Escherichia coli bacteria from which they cloned this protein. When sublingually applied to mice, it showed a significant increase in IgG antibody, which prevented the adhesion of $S$. mutans to the dental surfaces of the animals after vaccination [3]. 
On the other hand, Childers NK et al. have heretofore been the only ones to evaluate active vaccines for dental caries in human models, by administering a Streptococcus mutans antigen by the nasal or oral route. In this regard, the study by Childers NK et al. (2002), was applied to 21 adult volunteers, who were given a topical Streptococcus mutans vaccine on the tonsils and nasal surfaces in order to determine its effectiveness in inducing mucosal immune responses. This double-blind study tested two forms of the vaccine antigen, using soluble antigens and liposomal antigens, and used an enriched dose of streptococcal glucosyltransferase (E-GTF). The results of this study provide evidence of the safety of the enriched E-GTF preparation, both soluble and liposomal, in addition to nasal and amygdalin immunization for use in humans. Intranasal immunization generated a significantly higher mean anti-E-GTF IgA response, postimmunization, in nasal lavage and saliva samples obtained from the parotid, but not in serum of the patient, compared to the amygdalin immunization groups. In this same trial, subjects who received liposomal E-GTF had higher IgA responses compared to responses observed in individuals who received soluble E-GTF; however, the differences were not significant [21]. Childers' group concluded that immunization by the nasal route appears to be more effective than the oral route in achieving an anti-Streptococcus mutans mucosal response in adults.

Later, Childers et al. (2006), conducted a new study in which they used the intranasal route to immunize, two years later, individuals previously immunized by nasal and amygdalin and compare their responses with those of individuals who were immunized for the first time (control group). The aim was to determine the effectiveness of a booster immunization regimen in increasing mucosal response. The trial results showed that intranasal immunization used as a booster does not generate significantly different salivary IgA (sIgA) responses from groups that received a single immunization. The main benefit was obtained with the group initially immunized by intranasal route (IN), which after receiving the booster vaccination administered by the same route (IN), obtained significantly higher levels of serum IgA concerning the remaining groups evaluated (control, and booster group that was initially immunized by amygdalin route). These results indicate that the booster vaccination with an E-GTF dose only shows benefit, if the objective is to reach significant serum IgA levels. However, booster vaccination does not demonstrate significance in obtaining salivary IgA responses when compared to a single-dose vaccination schedule [1].

Currently, there are few clinical trials of active immunization as a preventive approach in dental caries, so more human studies are needed to evaluate the true scope of this approach, and to establish its true efficacy, dose, and period of protection.

Tables 1 and 2 break down the advantages and disadvantages of passive and active immunization approaches, as we understand them.

Table 1 Advantages and disadvantages of passive immunization (PI)

\begin{tabular}{|l|l|}
\hline \multicolumn{1}{|c|}{ PI: Advantages } & \multicolumn{1}{|c|}{ PI: Disadvantages } \\
\hline $\begin{array}{l}\text { It avoids the risk of side effects from active } \\
\text { immunization. }\end{array}$ & It does not induce the generation of immunological memory. \\
\hline $\begin{array}{l}\text { Potential for antibodies to interfere with the ability of } \\
\text { Streptococcus mutans to colonize tooth surfaces, and } \\
\text { limit the development of caries. }\end{array}$ & $\begin{array}{l}\text { it needs continuous administration to obtain lasting } \\
\text { proten against pathogens. }\end{array}$ \\
\hline $\begin{array}{l}\text { The IgY-based commercial product Ovalgen DC, purified } \\
\text { from egg yolk, has shown comparable efficacy to } \\
\text { probiotic strains in reducing salivary levels of the } \\
\text { cariogenic } S \text {. mutans. }\end{array}$ & $\begin{array}{l}\text { It has a lower degree of effectiveness, due to the half-life of } \\
\text { the antibodies being a short period of time. }\end{array}$ \\
\hline $\begin{array}{l}\text { The commercial product Ovalgen DC has shown in } \\
\text { clinical studies superiority over triclosan in reducing } \\
\text { salivary levels of } S \text {. mutans. }\end{array}$ & $\begin{array}{l}\text { Low accessibility, and limited usefulness as a public health } \\
\text { measure. }\end{array}$ \\
\hline
\end{tabular}


Table 2 Advantages and disadvantages of active immunization (AI)

\begin{tabular}{|l|l|}
\hline \multicolumn{1}{|c|}{ AI: Advantages } & \multicolumn{1}{c|}{ AI: Disadvantages } \\
\hline $\begin{array}{l}\text { It induces the endogenous production of salivary } \\
\text { antibodies and generates immunological memory. }\end{array}$ & $\begin{array}{l}\text { Further human and long-term studies are required to } \\
\text { establish its effectiveness. }\end{array}$ \\
\hline $\begin{array}{l}\text { It has been shown to be highly effective in animal } \\
\text { studies. }\end{array}$ & May cause side effects in tissues outside the oral cavity. \\
\hline $\begin{array}{l}\text { The results in humans are encouraging. Significant and } \\
\text { lasting responses (at least 2 years) in salivary sIgA levels } \\
\text { would appear to be obtained with a single application, } \\
\text { without the need for reinforcement. }\end{array}$ & Limited access to the vast majority of the population. \\
\hline $\begin{array}{l}\text { Studies in humans report obtaining immune responses } \\
\text { with easy-to-apply forms such as the use of sprays. }\end{array}$ & $\begin{array}{l}\text { The highest documented human response, based on salivary } \\
\text { sIgA versus } S \text {. mutans GTF, are achieved under intra-nasal } \\
\text { administration and with liposomal coverage. The latter } \\
\text { formulation means a higher cost. }\end{array}$ \\
\hline
\end{tabular}

\subsection{Limitations of immunization approaches to dental caries}

For several years, S. mutans have been considered the main causal agent of dental caries, so both diagnosis and therapy have been oriented towards this microorganism. However, recent studies have discovered a polymicrobial ecosystem in carious lesions, and $S$. mutans represents a small part of the bacterial community, representing only $0.1 \%$ of the dental plaque and 0.7 to $1.6 \%$ of all the microbiota found in carious lesions. In this way, it is established that consortiums formed by multiple microorganisms, initiate and expand the cavity by acting together. Therefore, antimicrobial therapies may not be effective in treating caries and other polymicrobial diseases [11].

Bacterial compositions vary between individuals and even within the same carious lesion, since the active bacteria in initial enamel lesions are different from those found in more advanced dentin cavities [5]. According to the study by Simon Soro et al. (2015), carious lesions of enamel had about 177 bacterial phylotypes, while carious lesions with dentin exposed to the oral cavity had 251 species, and "hidden" dentin cavities that had contact with the oral cavity through a small enamel lesion had 201 bacterial phylotypes [11].

Simon Soro et al. (2014) performed a polymerase chain reaction (PCR) amplification of ribonucleic acid (RNA) extracted from carious lesions of enamel and dentin, after reverse transcription. By performing amplification of the $16 \mathrm{~S}$ gene from RNA, active bacteria that contribute to the development of the carious lesion can be determined, as the amount of ribosomal RNA (rRNA) in the bacterial cells is associated with their degree of metabolic activity, while DNA-based studies have the drawback that the PCR step can amplify the DNA of inactive or even dead microorganisms. It was found that Lactobacillus were almost exclusively predominant in dentin lesions, while Streptococci represented $40 \%$ of the total active community in adamantine caries and $20 \%$ in dentin caries. Therefore, due to the high frequency of Streptococci, streptococcal sequences were identified at the species level (Fig. 1). Thus, Streptococcus sanguinis was the most representative type of Streptococci in dentine cavities, while Streptococcus mitis was more abundant in enamel cavities than in dentine lesions [5]. 


\section{Distribution of the three main species of Streptococcus detected in different caries lesions in the Spanish population.}
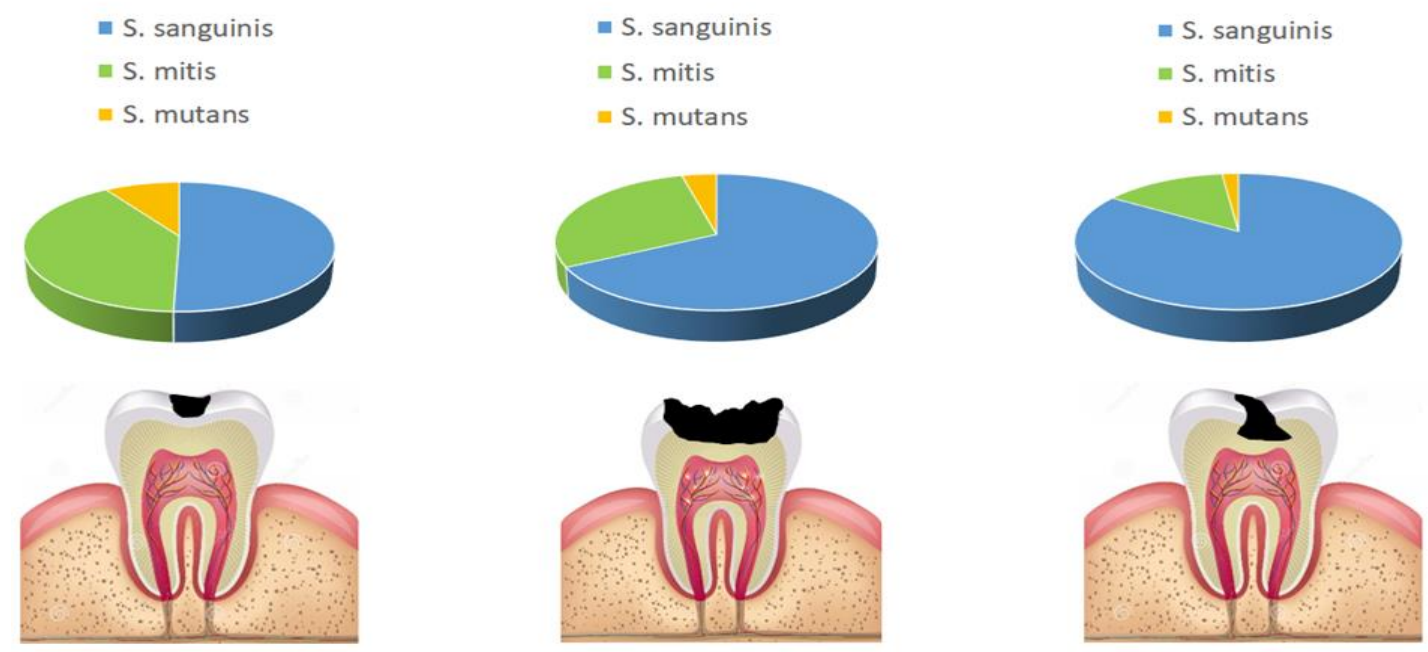

Figure 1. Proportion of different Streptococcus species in different types of carious lesions, according to findings of Simon Soro et al. (2014), in their study on composition of cariogenic microbiota as a function of the type of lesion, obtained by OMICAS approaches in Spanish subjects.

Streptococcus mutans, as an active microorganism of carious lesions, represents $0.73 \%$ in enamel cavities, $0.48 \%$ in open dentin cavities and $0.02 \%$ in hidden dentin cavities [5]. Therefore, although there are studies that determine the association between $S$. mutans and caries risk, other species emerge as major representatives in the adamantine cavity microbial community, including Veillonella spp. Rothia spp. and Leptotrichia spp. While S. sanguinis, Atopobium spp. Schlegelella spp. Pseudoramibacter spp. and Lactobacillus spp. have been isolated in dentine cavities [11]. Consequently, the low proportion of $S$. mutans detected, confirms that this species is in minority in the oral microbiota, and questions its role as the main etiological agent of dental caries [5].

In addition, the variation of bacterial compositions among individuals is reported, since Aas et al. (2008), found in the temporary dentition elevated levels of Corynebacterium spp. and Actinomyces gerencseriae, while in the permanent dentition Leptotrichia spp. was identified. Campylobacter gracilis, and Selenomonas spp. [6]. Due to the variation of bacterial consortia in the different stages of dental caries, types of dentition, and among individuals, it is hypothesized that different sets of microorganisms can perform the same functions. This is supported by genomic studies of the human microbiome, where different taxonomic compositions produce similar functional results [11].

In view of the above, we consider necessary to evaluate the long term effect of both active and passive immunization schemes, regarding their capacity to reduce the incidence of caries measured through indexes; and to consider the role of these approaches in combination with classical prevention strategies aimed at mechanical and chemical control of dental plaque, and low-carbohydrate diet management.

\subsection{Final considerations}

There are several approaches to dental caries prevention, some based on biofilm control and modulation methods that have failed to reduce the incidence of caries significantly; and others based on stimulating or providing humoral immune response against the major cariogenic phylotypes. Within this last group, active and passive immunization methods are recognized, which have been questioned in terms of efficacy, given the heterogeneity in the microbiology of dental caries.

Both approaches to immunization show advantages and disadvantages. Regarding passive immunization, the system that has shown in clinical studies superiority over other types of antibodies, is the system based on protection with chicken IgY, of which there are already commercial products. We consider that this system would be beneficial, as a measure of caries prevention, in immunosuppressed population with incapacity to generate antibodies actively, in patients with xerostomia, and as a measure of public health to the population with scarce economic resources to feed themselves properly (low carbohydrate diet), and to acquire elements of oral hygiene. 
So far, there are few clinical trials evaluating active immunization in dental caries. However, this approach has proven to be the most effective and durable method, due to the involvement of the host response, as antibodies developed against adhesives prevent the adhesion of Streptococcus mutans to tooth structure. These strategies include mucosal immunization, which results in salivary anti-S. mutans responses, with nasal immunization being the most effective route to induce mucosal responses. However, further human trials are needed to establish true efficacy in terms of reducing the incidence of dental caries, as well as the dose and period of protection.

It promises the genetic modification of habitual bacteria of the oral microbiota, to express antigens that originate the activation and synthesis of antibodies that can be united to several epitopes present in the surface of the cariogenic bacteria with greater predominance, as much in the carious lesions of enamel and dentine, as well as in different anatomical sites, ethnic groups and types of dentition. We consider that research should focus on an immunization regime that maximizes the induction, in the human host, of polyclonal/polyvalent antibodies in saliva, capable of neutralizing several classes of epitopes shared by the most prevalent bacteria in the cariogenic process, such as Streptococcus spp, and Lactobacillus spp.

Genomic or transcriptomic studies, by current sequencing platforms, are necessary to identify bacterial species associated with different types of carious lesions in the Ecuadorian population. This will allow us to recognize the bacterial taxa associated to dental caries with greater prevalence in our population, and thus design preventive approaches based on immunization strategies. Indeed, knowing the specific microbial composition of the carious process in our population is the first step in designing preventive strategies, whether they are based on modulation of oral biofilm and mucosal-associated immunity, or whether they are based on active immunization approaches.

\section{Conclusion}

Immunization against dental caries, by active or passive methods, represents an innovative and avant-garde way to undertake prevention programs and reduction in the incidence of dental caries; however, we consider that passive immunization, due to its disadvantages listed in this review, constitutes a measure of little use as a public health strategy; transferring its greatest benefit to very specific clinical cases. Therefore, we propose to consider passive immunization as a form of personalized oral medicine. For its part, active immunization, although it shows greater strengths compared to passive immunization, more research is needed to determine its real effectiveness in reducing the incidence of dental caries, as well as to reliably determine dose and safety. Evidence as of now suggests that liposome-coated glucosyltransferase and nasal administration are the most optimal way to deliver antigens from cariogenic bacteria, resulting in elevated levels of sIgA in saliva.

\section{Compliance with ethical standards}

\section{Acknowledgments}

Our fraternal thanks to our patients, who are the reason we continue learning and updating us.

\section{Disclosure of conflict of interest}

The authors declare that this article has not received funds from any entity, for its preparation and publication; and we have no conflicts of interest.

\section{References}

[1] Childers N, Li F, Dasanayake A, Li Y, Kirk K, Michalek S. Immune response in humans to a nasal boost with Streptococcus mutans antigens. Oral Microbiol Immunol. 2006; 21(5): 309-13.

[2] Negroni M. Microbiología estomatológica fundamentos y guía práctica. Editorial Médica Panamericana. 2017.

[3] Ferreira E, Batista M, Cavalcante R, Pegos V, Passos H, Silva D, et al. Sublingual immunization with the phosphatebinding-protein (PstS) reduces oral colonization by Streptococcus mutans. Mol Oral Microbiol. 2016; 31(5): 41022. 
[4] Cao X, Fan J, Chen J, Li Y, Fan M. Immunogenicity and prediction of epitopic region of antigen Ag I/II and glucosyltransferase from Streptococcus mutans. J Huazhong Univ Sci Technol - Med Sci. 2016; 36(3): 416-21.

[5] Simón-Soro A, Guillen-Navarro M, Mira A. Metatranscriptomics reveals overall active bacterial composition in caries lesions. J Oral Microbiol. 2014; 6(1).

[6] Aas J, Griffen A, Dardis S, Lee A, Olsen I, Dewhirst F, et al. Bacteria of dental caries in primary and permanent teeth in children and young adults. J Clin Microbiol. 2008; 46(4): 1407-17.

[7] Bascones A, Morante S. Antisépticos orales. Revisión de la literatura y perspectiva actual. Implant Av Periodon. 2006; 31-59.

[8] Ghasempour M, Sefdgar S, Moghadamnia A, Ghadimi R, Gharekhani S, Shirkhani L. Comparative study of Kefir yogurt-drink and sodium fluoride mouth rinse on salivary mutans streptococci. J Contemp Dent Pract. 2014; 15(2): 214-7.

[9] Cogulu D, Topaloglu-Ak A, Caglar E, Sandalli N, Karagozlu C, Ersin N, et al. Potential effects of a multistrain probiotic-kefir on salivary Streptococcus mutans and Lactobacillus spp. J Dent Sci [Internet]. 2010; 5(3): 144-9.

[10] James F, Butler C, Adams G, Mitchell L, Dashper S, Escobar K, et al. The Prebiotic Effect of CPP-ACP Sugar-Free Chewing Gum. J Dent [Internet]. 2019; 103225.

[11] Simón-Soro A, Mira A. Solving the etiology of dental caries. Trends Microbiol. 2015; 23(2): 76-82.

[12] Kassebaum N, Smith A, Bernabé E, Fleming T, Reynolds A, Vos T, et al. Global, Regional, and National Prevalence, Incidence, and Disability-Adjusted Life Years for Oral Conditions for 195 Countries, 1990-2015: A Systematic Analysis for the Global Burden of Diseases, Injuries, and Risk Factors. J Dent Res. 2017; 96(4): 380-7.

[13] Nishihara T, Suzuki N, Yoneda M, Hirofuji T. Effects of Lactobacillus salivarius -containing tablets on caries risk factors : a randomized open-label clinical trial. BMC Oral Health. 2014; 14(1): 1-7.

[14] Van Sa N. Specific Egg Yolk Antibody (Ovalgen DC) as a Novel Supportive Immunotherapy for Dental Caries. Immunol Res Inst Gifu. 2013; 1-29.

[15] Aravindha B, Malathi L, Karthick R, Leena Sankari S. Immunology of dental caries. Biomed Pharmacol J. 2016; 9(2): 823-6.

[16] Costalonga M, Herzberg M. The oral microbiome and the immunobiology of periodontal disease and caries. Immunol Lett [Internet]. 2014; 162(2): 22-38.

[17] Smith D, King W, Rivero J, Taubman M. Immunological and protective effects of diepitopic subunit dental caries vaccines. Infect Immun. 2005; 73(5): 2797-804.

[18] Shimazaki Y, Mitoma M, Oho T, Nakano Y, Yamashita Y, Okano K, et al. Passive immunization with milk produced from an immunized cow prevents oral recolonization by Streptococcus mutans. Clin Diagn Lab Immunol. 2001; 8(6): 1136-9.

[19] Culshaw S, LaRosa K, Tolani H, Han X, Eastcott J, Smith D, et al. Immunogenic and protective potential of mutans streptococcal glucosyltransferase peptide constructs selected by major histocompatibility complex class II allele binding. Infect Immun. 2007; 75(2): 915-23.

[20] Robinette R, Oli M, McArthur W, Brady J. A therapeutic anti-Streptococcus mutans monoclonal antibody used in human passive protection trials influences the adaptive immune response. Vaccine [Internet]. 2011; 23(1): 1-7.

[21] Childers N, Tong G, Li F, Dasanayake A, Kirk K, Michalek S. Humans Immunized with Streptococcus mutans Antigens by Mucosal Routes. J Dent Res. 2002; 81(1): 48-52.

[22] Smith D. Prospects in caries vaccine development. J Dent Res. 2012; 91(3): 225-6.

[23] Mitoma M, Oho T, Michibata N, Okano K, Nakano Y, Fukuyama M, et al. Passive immunization with bovine milk containing antibodies to a cell surface protein antigen-glucosyltransferase fusion protein protects rats against dental caries. Infect Immun. 2002; 70(5): 2721-4.

[24] Bachtiar E, Soejoedono R, Bachtiar B, Henrietta A, Farhana N, Yuniastuti M. Effects of soybean milk, chitosan, and anti-Streptococcus mutans IgY in malnourished rats' dental biofilm and the IgY persistency in saliva. Interv Med Appl Sci. 2015; 7(3): 118-23.

[25] Krüger C, Hultberg A, Van Dollenweerd C, Marcotte H, Hammarström L. Passive immunization by lactobacilli expressing single-chain antibodies against Streptococcus mutans. Mol Biotechnol. 2005; 31(3): $221-31$. 


\section{Author's short biography}

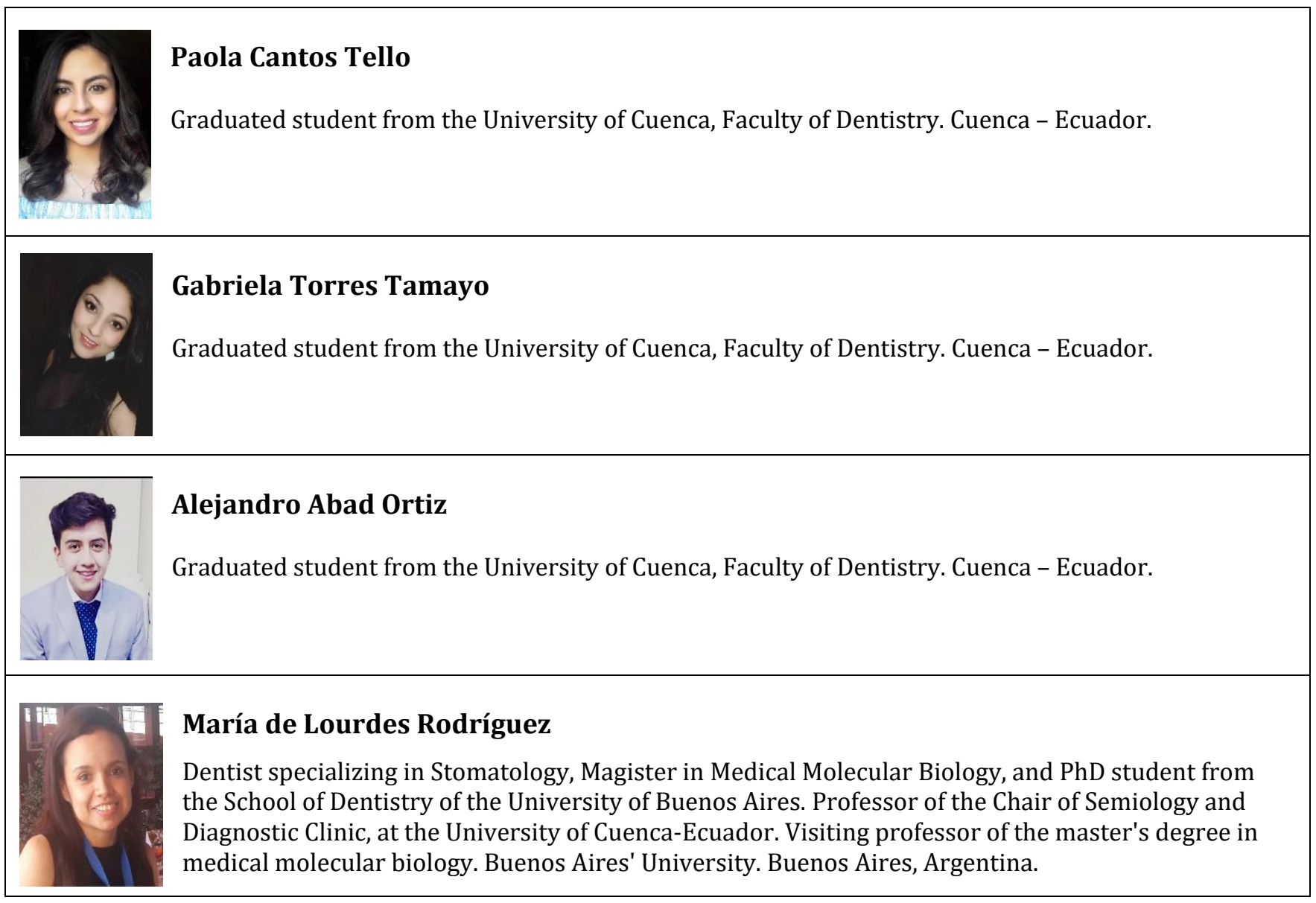

